A review of the dynamics of cavitating pumps

This article has been downloaded from IOPscience. Please scroll down to see the full text article.

2012 IOP Conf. Ser.: Earth Environ. Sci. 15012001

(http://iopscience.iop.org/1755-1315/15/1/012001)

View the table of contents for this issue, or go to the journal homepage for more

Download details:

IP Address: 131.215.225.160

The article was downloaded on 19/07/2013 at 21:02

Please note that terms and conditions apply. 


\title{
A review of the dynamics of cavitating pumps
}

\author{
C E Brennen \\ Hayman Professor of Mechanical Engineering, Emeritus, California Institute of \\ Technology, Psadena, California 91125, USA \\ brennen@caltech.edu
}

\begin{abstract}
This paper presents a review of some of the recent developments in our understanding of the dynamics and instabilities caused by cavitation in pumps. Focus is placed on presently available data for the transfer functions for cavitating pumps and inducers, particularly on the compliance and mass flow gain factor which are so critical for pump and system stability. The resonant frequency for cavitating pumps is introduced and contexted. Finally emphasis is placed on the paucity of our understanding of pump dynamics when the device or system is subjected to global oscillation.
\end{abstract}

\section{Introduction}

Since the first experimental measurements many years ago of the complete dynamic transfer function for a cavitating pump ( $\mathrm{Ng}$ and Brennen 1976, Brennen et al. 1982) there has been a general recognition of the importance of various components of these transfer functions (particularly the cavitation compliance and mass flow gain factor) in determining the dynamic characteristics and instabilities of systems incorporating such pumps (see for example Rubin 1966 \& 1970, Oppenheim and Rubin 1993, Tsujimoto et al. 2001, Dotson et al. 2005). The present paper attempts to summarize some of the recent understandings and to evaluate the current state of knowledge of transfer functions for cavitating pumps..

\section{Pump transfer function data}

The linear dynamic transfer matrix for a pump is denoted here by $\mathrm{TP}_{\mathrm{ij}}$ and is defined by

$$
\left\{\begin{array}{c}
P_{2} \\
m_{2}
\end{array}\right\}=\left[\begin{array}{ll}
T P_{11} & T P_{12} \\
T P_{21} & T P_{22}
\end{array}\right]\left\{\begin{array}{c}
P_{1} \\
m_{1}
\end{array}\right\}
$$

where $\mathrm{P}$ and $\mathrm{m}$ are the complex, linearized fluctuating total pressure and mass flow rate and subscripts 1 and 2 refer to the pump inlet and discharge respectively. In general $\mathrm{TP}_{\mathrm{ij}}$ will be a function of the frequency, $\omega$, of the perturbations and the mean flow conditions in the pump including the design, the cavitation number, $\sigma$, and the flow coefficient. In this review we will focus primarily on the second of these equations and on $\mathrm{TP}_{21}$ and $\mathrm{TP}_{22}$ since cavitation has a major effect on these characteristics and they therefore have a critical influence on the potential instabilities in the fluid system in which the pump is installed. But it is valuable in passing to note that $T_{12}=-R-j \omega L$ where $R$ is the pump resistance and $\mathrm{L}$ is the pump inertance (valuable measurements of these dynamic characteristics for a non-cavitating pump were first made by Ohashi, 1968, and by Anderson et al., 1971). In the absence of cavitation and compressibility effects $\mathrm{TP}_{11}=1$ but its departure from unity due to cavitation is also important in pump dynamics. 
The transfer function and other pump dynamic characteristics presented in this paper are nondimensionalized in the manner of Brennen et al. (1982). Specifically the frequency, $\omega$, is nondimensionalized as $\omega^{\prime}=\omega \mathrm{h} / \mathrm{U}_{\mathrm{t}}$ where $\mathrm{h}$ is the peripheral blade tip spacing at the inlet to the pump or inducer ( $h=2 \pi R_{t} / N$ where $R_{t}$ is the inlet tip radius and $N$ is the number of main blades) and $U_{t}$ is the inlet tip speed $\left(\mathrm{U}_{\mathrm{t}}=\Omega \mathrm{R}_{\mathrm{t}}\right.$ where $\Omega$ is the rotational speed in rad/s). Then the compliance, C, and mass flow gain factor, $\mathrm{M}$, are defined by expanding the transfer function elements, $\mathrm{TP}_{21}$ and $\mathrm{TP}_{22}$, at low frequency in a power series in $\mathrm{j} \omega$ :

$$
\begin{gathered}
T P_{21}=-j \omega C+(j \omega)^{2} C^{*}+\ldots \\
T P_{22}=1-j \omega M+(j \omega)^{2} M^{*}+\ldots
\end{gathered}
$$

The compliance, $\mathrm{C}$, and mass flow gain factor, $\mathrm{M}$, are non-dimensionalized by

$$
\frac{C N \Omega^{2}}{4 \pi^{2} R_{t}} \text { and } \frac{M N \Omega}{2 \pi}
$$

Note that the above non-dimensionalization scheme differs from that used in Brennen (1994) but is preferred since each blade produces cavitation that contributes to $\mathrm{C}$ and $\mathrm{M}$.

Those first experimental measurements of the complete dynamic transfer function for a cavitating pump (Ng and Brennen 1976, Brennen et al. 1982) were carried out in water with a series of model inducers including a scale model of the low pressure LOX inducer in the Space Shuttle Main Engine (SSME). A typical photograph of the $10.2 \mathrm{~cm}$ diameter version of that inducer under moderate cavitating conditions is included as Figure 1. which illustrates the tip clearance backflow and cavitation that is typical of many inducers (Brennen 1994).

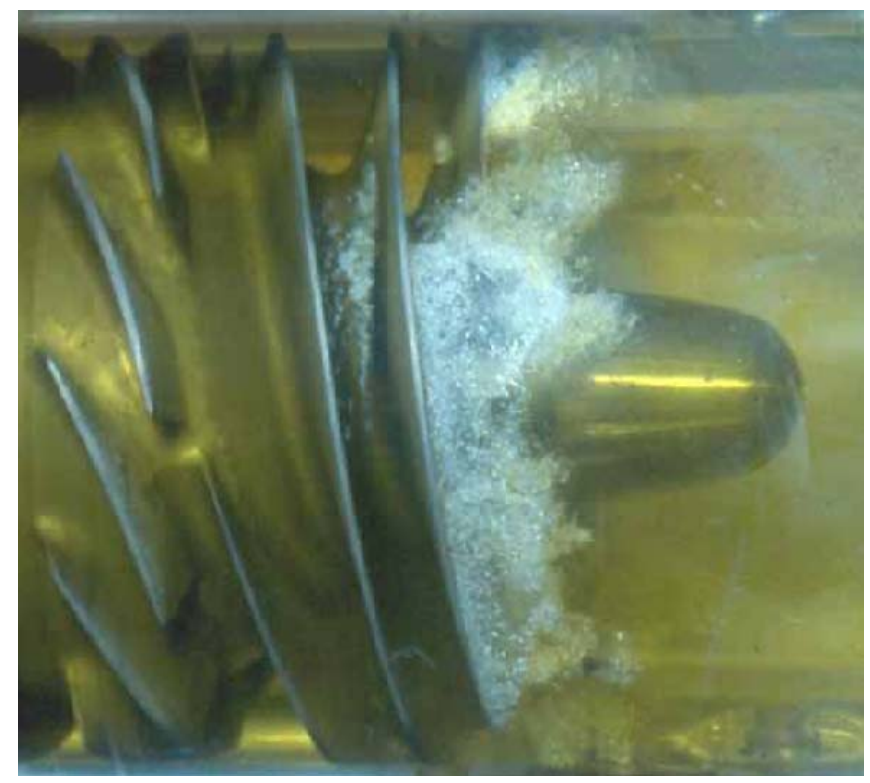

Figure 1. Scale model of the low pressure liquid oxygen pump impeller for the Space Shuttle Main Engine (SSME) in moderate cavitating conditions in water.

Measured transfer functions for that $10.2 \mathrm{~cm}$ diameter SSME inducer operating in water at $6000 \mathrm{rpm}$, a flow coefficient of $\phi_{1}=0.07$ and various cavitation numbers, $\sigma$, are reproduced in Figure 2. (left) where the four transfer functions elements are each plotted against a dimensionless frequency, the real parts as the solid lines and the imaginary parts as dashed lines. We should note that this data necessarily has substantial uncertainity associated with it and therefore polynomial fits in the Laplace variable j $\omega$ were produced in order to extract quantities like R, L, C and M (the polynomial fits to Figure 2. (left) are shown on Figure 2. (right)). An up-to-date collection of the available data on the compliance and the mass flow gain factor is presented in Figure 3. where those quantities are plotted 
against the cavitation number. The data on the SSME inducers in water is extracted from Figure 2. while the J2 oxidizer data was derived by Brennen and Acosta (1976) using test data and a heuristic dynamic model of the test facility. The LE-7 test data in liquid nitrogen was obtained by Shimura (1995). The LE-7A data is the only LOX data and was also extracted from test data by Hori and Brennen (2011). All of this data is subject to significant uncertainty though the original SSME data is probably the most reliable since it is based on measurements of the complete dynamic transfer function. Nevertheless, with one exception, both the compliance and mass flow gain factor data exhibit significant consistency in which both $\mathrm{C}$ and $\mathrm{M}$ are inversely proportional to $\sigma$. The exception is the LE-7A LOX data for the mass flow gain factor; whether this discrepancy is within the uncertainty band or an actual LOX thermal effect remains to be seen.
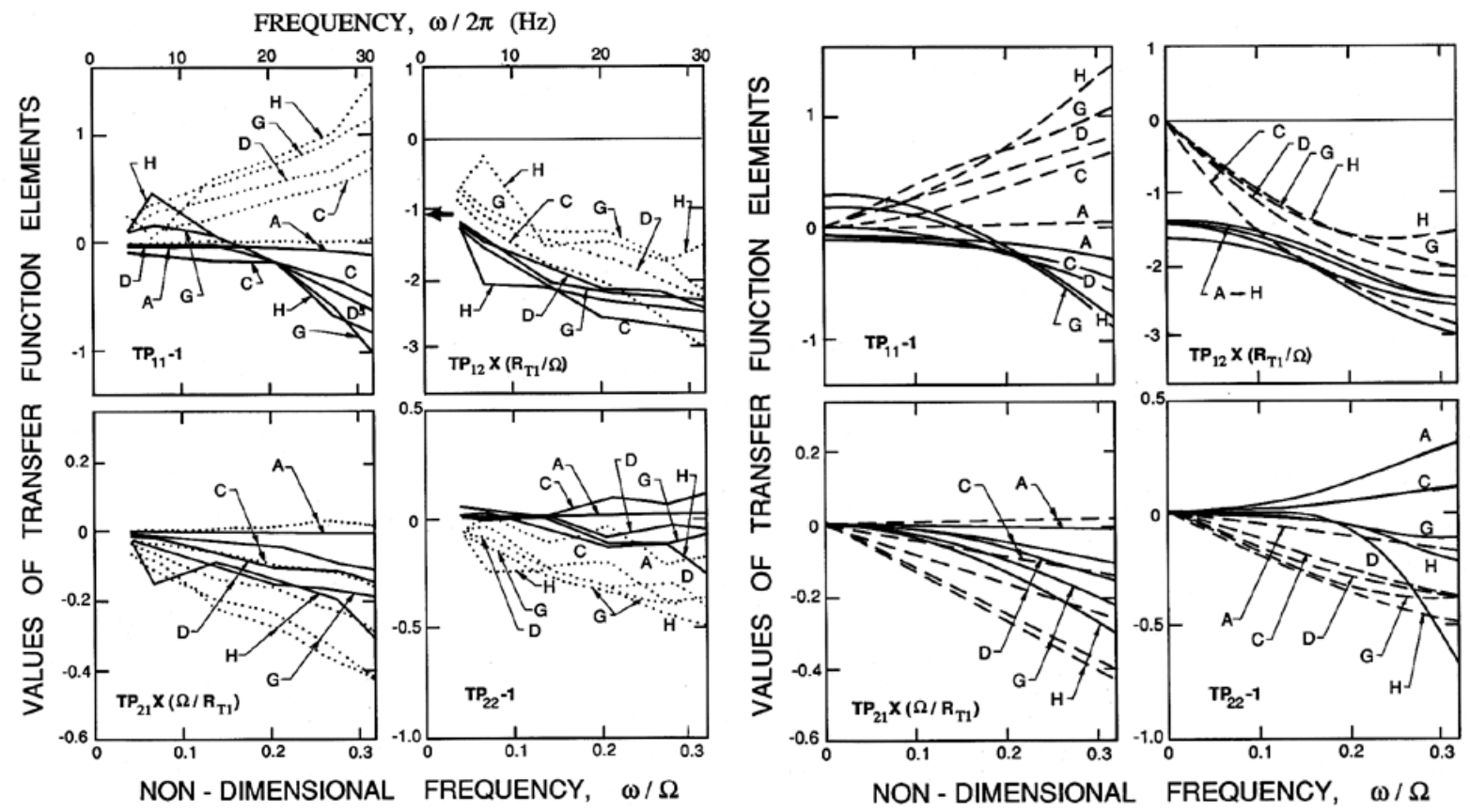

Figure 2. Left: Typical transfer functions for a cavitating inducer obtained by Brennen et al. (1982) for the $10.2 \mathrm{~cm}$ diameter SSME inducer operating in water at 6000rpm and a flow coefficient of $\phi_{1}=0.07$. Data is shown for four different cavitation numbers, $\sigma=(A) 0.37$, (C) 0.10 , (D) $0.069,(G)$ 0.052 and $(\mathrm{H})$ 0.044. Real and imaginary parts are denoted by the solid and dashed lines respectively. The quasistatic pump resistance is indicated by the arrow. Right: Polynomial curves fitted to the data on the left. Adapted from Brennen et al. (1982).

Before further discussion of this data collection we digress briefly to introduce a property in the dynamics of cavitating pumps that has not received sufficient attention in the past, namely the fundamental resonant frequency of a cavitating pump.

\section{Resonant frequency of a cavitating pump}

It has been known for a long time that a cavitating inducer or pump may exhibit a violent surge oscillation at subsynchronous frequencies that results in very large pressure and flow rate oscillations in the system of which the pump is a part (Sack and Nottage 1965, Rosemann 1965, Natanzon et al. 1974, Miller and Gross 1967, Braisted \& Brennen 1980, Brennen 1994, Zoladz 2000). In the early days, this was known as "auto-oscillation" but the preferred name in recent times has been "cavitation surge". It typically occurs at low cavitation numbers just above those at which cavitation head loss becomes severe. Often it is preceded by a rotating cavitation pattern (see, for example, Kamijo et al. 
1994, Tsujimoto et al. 1993, Hashimoto et al. 1997, Zoldaz 2000). Figure 4. reproduces data on the frequencies of oscillation observed for the model SSME inducer and for a helical inducer by Braisted and Brennen (1980); they also plotted a rough empirical fit to that data which approximated the dimensionless surge frequency by $(5 \sigma)^{1 / 2}$. More recently we recognize that this "natural frequency of a cavitating pump" has a more fundamental origin as follows:

Almost any reasonable, proposed dynamic model for a cavitating inducer or pump (such as that on the right of Figure 4. designed to simulate the parallel streams of main flow and tip clearance flow) which incorporates both the pump inertance, L, and the cavitation compliance, C, clearly exhibits a natural frequency, $\Omega$ p , given by

$$
\Omega_{P}=\frac{1}{(L C)^{\frac{1}{2}}}
$$

Using the data for the SSME LOX inducer from Brennen (1994) we can approximate L and C by

$$
L \approx \frac{10}{R_{t}} \text { and } C \approx \frac{0.05 R_{t}}{\sigma \Omega^{2}}
$$

so that, substituting into Equation 5.,

$$
\frac{\Omega_{P}}{\Omega} \approx(2 \sigma)^{\frac{1}{2}} \quad \text { or } \quad \Omega_{P}^{\prime} \approx \frac{\Omega_{P} h}{U_{t}} \approx(5 \sigma)^{\frac{1}{2}}
$$

This is precisely the same as the result proposed empirically by Braisted and Brennen (1980) and shown on the left in Figure 4. We will refer to this as the natural frequency of a cavitating pump. Indeed the data of Figure 4.(left) displays further detail of this cavitating pump property. There is a manifest trend for the frequency to decrease somewhat with flow coefficient and this seems certain to be the result of an increasing volume of cavitation and increasing compliance as the blades are loaded up at lower flow coefficients. It is important to emphasize that this does not necessarily mean that the major system instability oscillations occur at this frequency. The study of Hori and Brennen (2011) discussed later in this paper shows, however, that major instabilities or resonances can occur when this natural frequency for a cavitating pump coincides with other system frequencies such as an organ pipe mode in a suction or discharge tube.
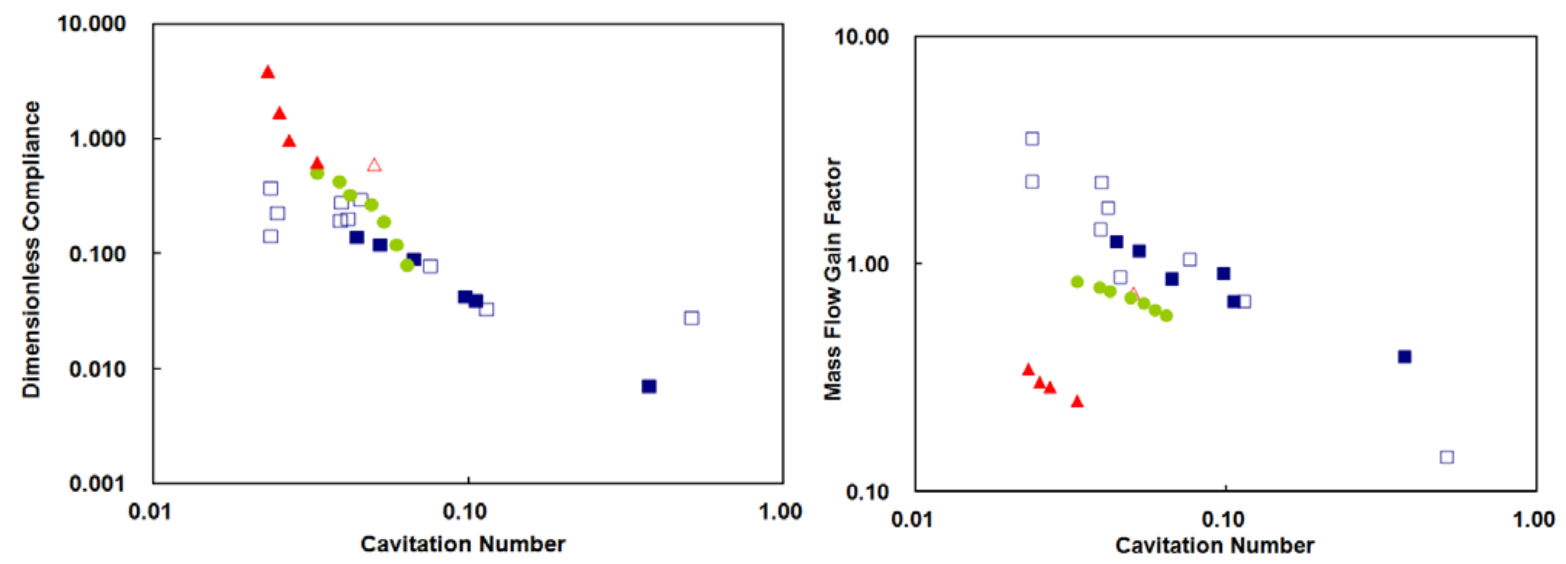

Figure 3. Dimensionless cavitation compliance (left) and mass flow gain factor (right) plotted against tip cavitation number for: [a] Brennen et al. (1982) SSME 10.2cm model inducer in water (solid blue squares) [b] Brennen et al. (1982) SSME 7.6cm model inducer in water (open blue squares) [c] Brennen \& Acosta (1976) J2-Oxidizer (solid green circles) analysis [d] Hori \& Brennen (2011) LE-7A LOX data (solid red triangles) [e] Shimura (1995) LE-7 LN2 data (open red triangles). 

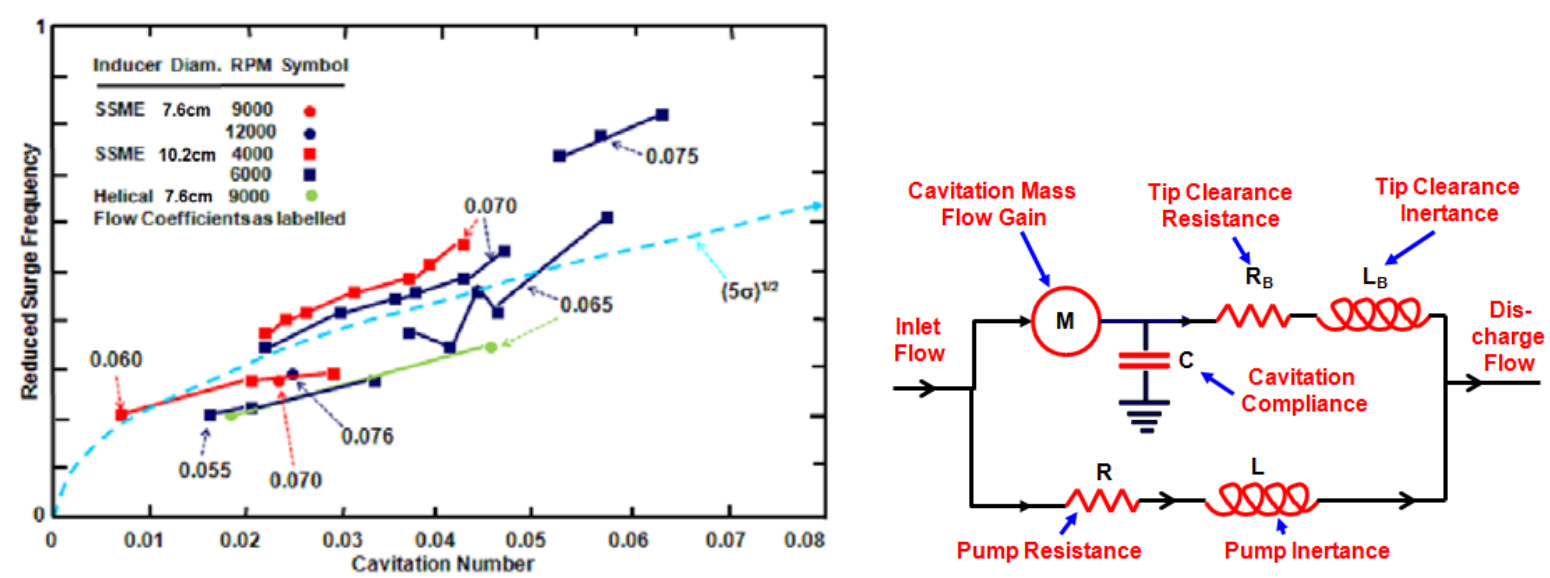

Figure 4. Left: Non-dimensional cavitation surge frequency as a function of cavitation number for the SSME model inducers at various speeds and flow coefficients as shown. The theoretical prediction is the dashed blue line, $(5 \sigma)^{1 / 2}$. Adapted from Braisted and Brennen (1980). Right: A dynamic model of the main flow and the parallel tip clearance backflow in a cavitating inducer.

\section{Phase lags in the cavitation dynamics}

Several researchers (Brennen 1973, Otsuka et al. 1996, Rubin 2004) have pointed out that the compliance and mass flow gain factor may become complex as the frequency increases and that this can have important consequences for launch vehicles. This is clearly equivalent to significant values of the quadratic terms in the expansion of Equations 1. and 2. but Rubin puts the values of $\mathrm{C}^{*}$ and $\mathrm{M}^{*}$ in terms of a compliance phase lag and a mass flow gain factor phase lag. One can visualize these phase lags as delays in the cavitation volume response to the pressure and incidence angle perturbations respectively. In this paper we will follow Rubin in writing the expansions of Equations 1. and 2. up to and including the quadratic order as

$$
\begin{gathered}
T P_{21}=-j \omega C\left\{1-j \omega^{\prime} \tau_{C}\right\} \\
T P_{22}=1-j \omega M\left\{1-j \omega^{\prime} \tau_{M}\right\}
\end{gathered}
$$

where $\tau_{\mathrm{C}}$ and $\tau_{\mathrm{M}}$ are the non-dimensional compliance phase lag and mass flow gain factor phase lag respectively. Data on these quadratic terms in the frequency expansions is, of course, subject to even greater uncertainity that the linear terms that lead to the compliance and mass flow gain factor. Nevertheless, in the light of the increasingly apparent importance of these terms, we have extracted values of $\tau_{\mathrm{C}}$ and $\tau_{\mathrm{M}}$ from the data of Figure 2. (right) and plotted them against cavitation number in Figure 5.

It may be valuable to make some tentative suggestions regarding these phase lags. It seems physically reasonable to envisage that a stream of cavitating bubbles (for example that carried forward by the backflow) would not respond immediately to the inlet pressure and flow rate fluctuations but would exhibit a phase lag delay that would increase with the frequency of the perturbations. Brennen (1973) investigated the compliance of a simple stream of cavitating bubbles at various frequencies, cavitation numbers and cavitation nuclei sizes. Figure 6. reproduces several figures from that paper which show that the compliance becomes increasingly complex as the frequency of the perturbations increases, and that the negative imaginary parts of the compliance which develop as the frequency increases represent just the kind of phase lag that we are addressing here (the magnitudes of the compliance in Figure 6. are not relevant to the current discussion). It is particularly interesting to observe that the reduced frequency plotted horizontally is defined as $f L_{s} / U_{s}$ where $f$ is the perturbation frequency (in $\mathrm{Hz}$ ) and $\mathrm{L}_{s}$ and $\mathrm{U}_{\mathrm{s}}$ are respectively the length and velocity of the simple stream of cavitating bubbles studied. Note from Figure 6. that the phase lag becomes important when the 
reduced frequency increases beyond a value of about 0.1 . Note, also, that the frequency, $f L_{s} / U_{s}$ of unity is a kinematic frequency associated with the entry and exit of bubbles from the cavitating zone rather than a dynamic frequency associated with the oscillation of the cavitation volume.

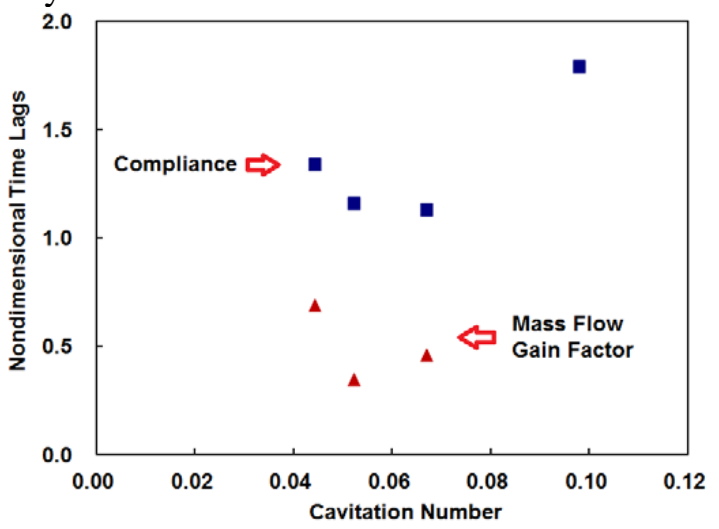

Figure 5. Non-dimensional time lags for the compliance, $\tau_{\mathrm{C}}$, and the mass flow gain factor, $\tau_{\mathrm{M}}$, as functions of the cavitation number for the SSME $10.2 \mathrm{~cm}$ model inducer in water. Taken from the data of Brennen et al. (1982).

Note that the uncertainties in this data probably exceed $50 \%$. Nevertheless we might suggest that the phase lags appear to be roughly independent of the cavitation number and to be somewhat greater for the compliance than for the mass flow gain factor.
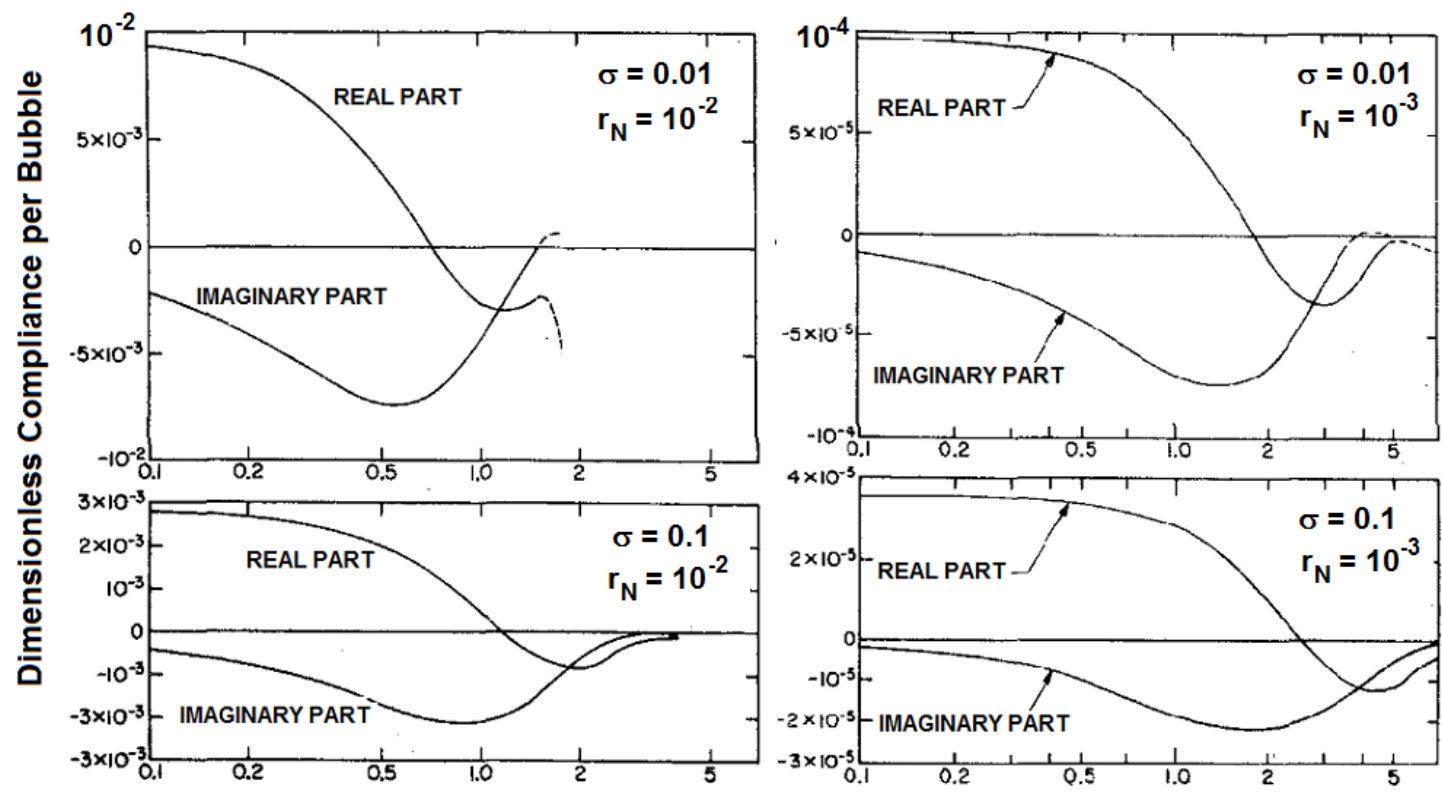

Reduced Frequency, $\omega \mathrm{L}_{\mathrm{s}} / 2 \pi \mathrm{U}_{\mathrm{s}}$

Figure 6. Real and imaginary parts of the dimensionless compliance (per bubble) of a stream of cavitating bubbles as functions of a reduced frequency based on the length of the cavitation zone, $\mathrm{L}_{\mathrm{s}}$, and its typical velocity, $\mathrm{U}_{\mathrm{s}}$. Results shown for several cavitation numbers, $\sigma$, and bubble nuclei size, $\mathrm{r}_{\mathrm{N}}$.

From Brennen (1973).

Let us consider the corresponding reduced frequency for the backflow cavitation in the experiments of Brennen et al. (1982) and Figure 2. For a 10.2 cm diameter inducer at a speed of 6000 rpm, a flow 
coefficient of $\phi_{1}=0.07$ (so that $U_{s}$ is approximately $200 \mathrm{~cm} / \mathrm{s}$ ) and an estimate length $L_{s}$ of about $10 \mathrm{~cm}$ the frequency that corresponds to a value of unity for $f L_{s} / U_{s}$ is $f=20 H z$. This corresponds well to the frequency in Figure 2. at which the imaginary parts of the compliance are observed to become well developed. However, this proposed physical explanation of the compliance phase lag also has some worrying implications. It suggests that the scaling of the phase lags may be a cause for concern for, at much higher rotational speeds, the phase lag would be much smaller and, consequently, any stability benefit that might accrue from it would be much smaller. However, in the absence of any hard evidence for the scaling of these quadratic effects, all we can conclude at present is that more measurements over a broader range of rotational speeds is needed in order to establish appropriate scaling for the phase lags.

We should note before leaving this topic that Otsuka et al. (1996) show that a blade cavitation model can also yield complex compliances and mass flow gain factors that correspond to time lags qualitatively similar to those presented in Figure 5.

\section{Comments on some analytical models}

We comment in the conclusions on the difficulties with any detailed CFD approach that aims to predict the dynamic transfer function for a cavitating inducer. It seems clear that much progress in developing reduced order models for cavitation in the complex geometry of an inducer (and, in particular, for the backflow cavitation) will be needed before this approach will provide practical and useful guidance. However, in the short term crude, one-dimensional models and lumped parameter models (see, for example, Cervone et al. (2009) guided by the existing data base can give useful benchmarks. The bubbly flow model of Brennen (1978) (see Figure 7. (left)) incorporated several of the basic phenomena that we now know are inherent in the dynamic response of an inducer or pump. In particular, the compliance of the bubbly stream within the flow (though the compressibility of that bubbly flow had to be represented by a empirical constant, $K^{\prime}$ ) and the magnitude of the void fraction fluctuations produced by the fluctuating angle of attack (represented by a second empirical factor of proportionality, M'). These two features respectively lead to dynamic waves and to kinematic waves in the bubbly blade passage flow. A typical transfer function derived from the bubbly flow model is reproduced in Figure 7. (right) and the similarity with the transfer functions in Figure 2. (right) is encouraging even though the two constants K' and M' were empirically chosen.
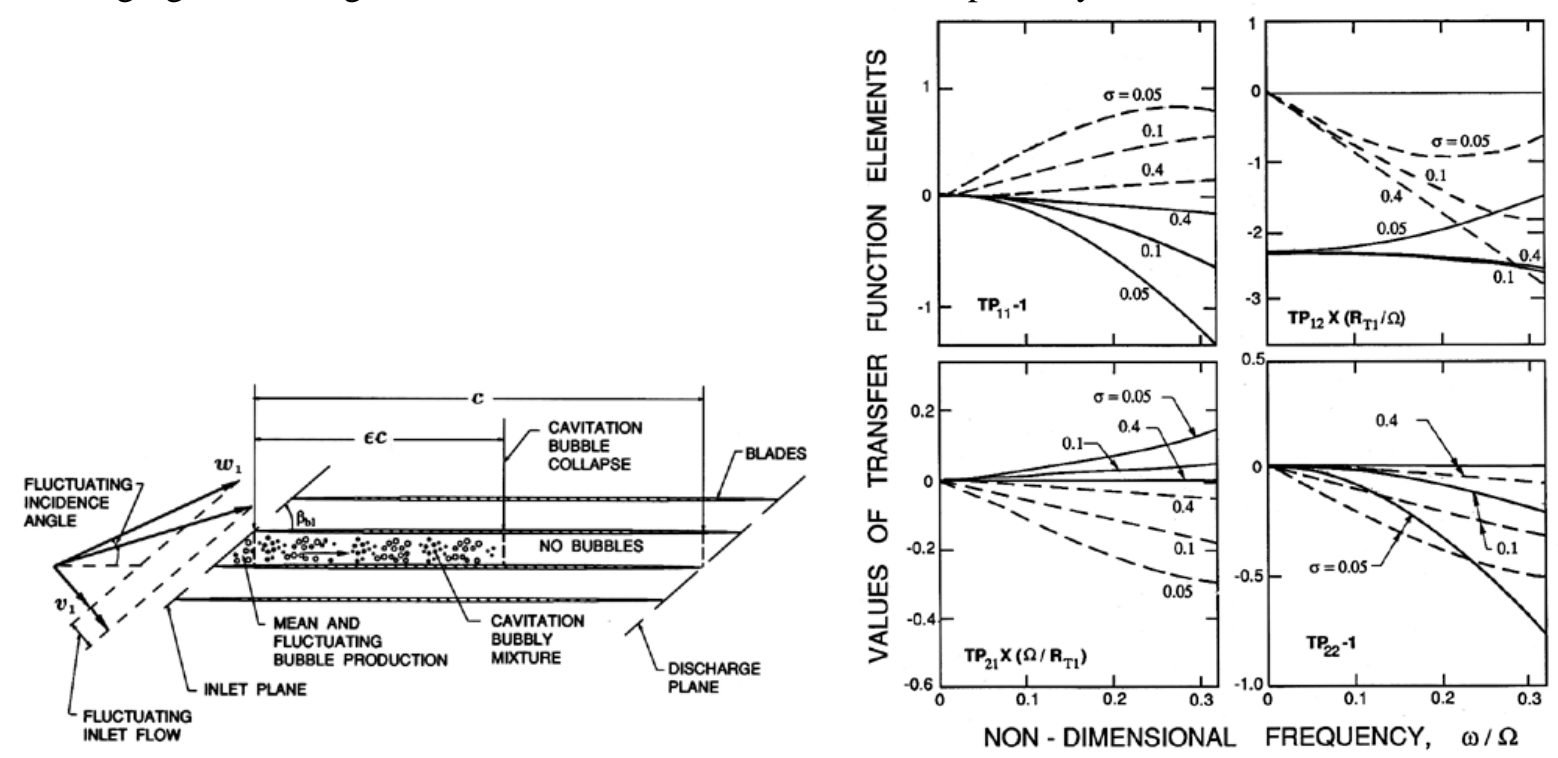

Figure 7. Left: Schematic of the bubbly flow model for the dynamics of cavitating pumps. Right: Transfer functions for the SSME inducer at $\phi_{1}=0.07$ calculated from the bubbly flow model. Adapted from Brennen (1978). 
The measured compliances and mass flow gain factors for the SSME inducers and for the J2 oxidizer inducer are reproduced in Figure 8. in order to compare that data with several predictions from the bubbly flow model (dashed blue lines for several choices of $\mathrm{K}^{\prime}$ and $\mathrm{M}^{\prime}$ ). The predictions appear to provide a useful benchmark for future data evaluation and comparison. Figure 8. also includes predictions from the blade cavitation analysis presented earlier by Brennen and Acosta (1976). That analysis has the advantage that it does not contain any empirical parameter, as such. However, it assumes that all the cavitation is contained within a single cavity attached to each blade. Moreover the comparisons in Figure 8. suggest that such a model does not yield very useful results which is not surprising when photographs such as Figure 1. indicate that the cavitation is primarily bubbly cavitation and not blade cavitation (Brennen 1994). Also included in Figure 8. are some quasistatic compliances and mass flow gain factors very recently derived by Yonezawa et al. (2012) from steady CFD calculations of the cavitating flow in linear cascades. They have also performed calculations at a series of flow coefficients that show a general trend of increasing compliance and mass flow gain factor as the flow coefficient is decreased.
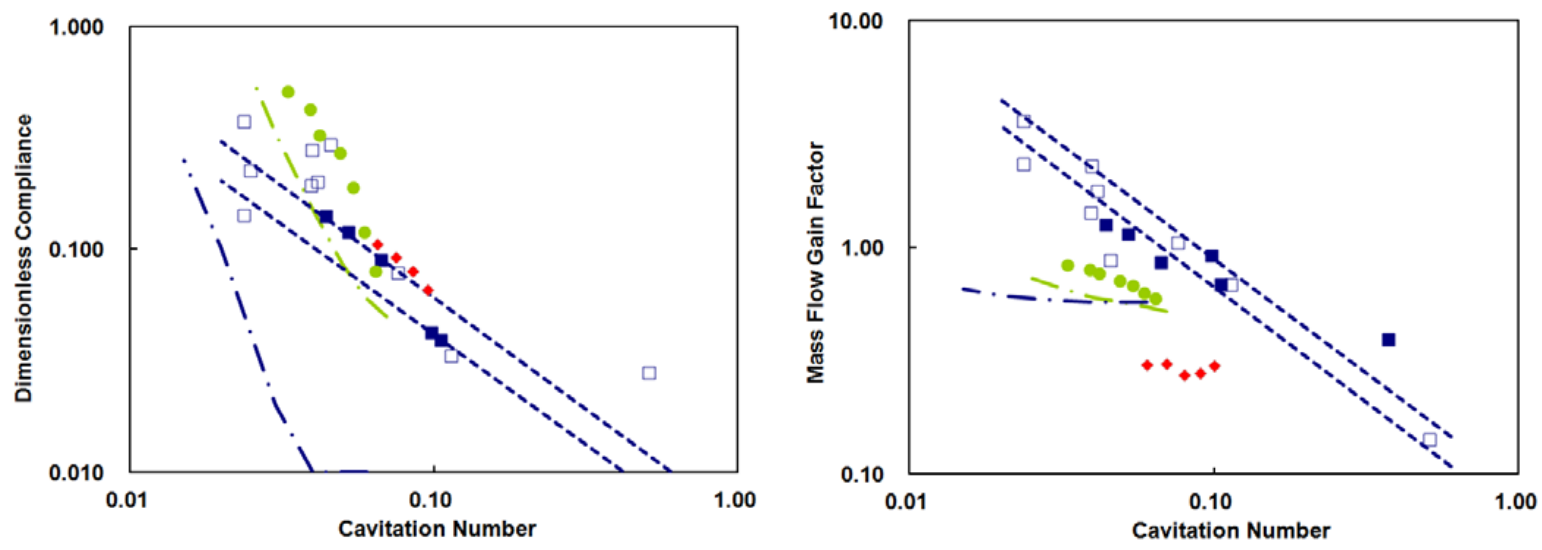

Figure 8. Dimensionless cavitation compliance (left) and mass flow gain factor (right) plotted against tip cavitation number for: [a] Brennen et al. (1982) SSME 10.2cm model inducer in water (solid blue squares) [b] Brennen et al. (1982) SSME 7.6cm model inducer in water (open blue squares) [c]

Brennen (1978) bubbly flow model results (dashed blue lines) [d] Brennen \& Acosta (1976) SSME

LPOTP blade cavitation prediction (dot-dash blue line) [e] Brennen \& Acosta (1976) J2-Oxidizer data (solid green circles) [f] Brennen \& Acosta (1976) J2-Oxidizer blade cavitation prediction (dot-dash green line) [g] Yonezawa et al. (2012) quasistatic CFD cascade data (solid red diamonds).

\section{Resonances in globally oscillating systems}

The research literature clearly exhibits a strong bias toward investigations of flow instabilities in systems which are essentially at rest, usually in a research laboratory test stand. While this bias is understandable, it can be misleading for it tends to mask the difference between such a flow instability and the resonant response in a flow system subject to global fluctuation. This is particularly an issue with launch vehicle propulsion systems for they can exhibit some serious resonances with the oscillating vehicle structure. Following the approach originally developed by Rubin (1966), Hori and Brennen (2011) recently constructed a time-domain model for prototypical pumping systems in order to examine the response of those systems to globally imposed acceleration, a(t). We review those results here for they present a case in which the static ground based systems appear free of serious instability but the same system exhibits serious resonance when subjected to global oscillation.

Hori and Brennen (2011) constructed dynamic models for four different configurations used during the testing and deployment of the LOX turbopump for the Japanese LE-7A rocket engine. As sketched in Figure 9., these configurations include three ground-based facilities, two cold-test facilities (one with a suction line accumulator and the other without), and a hot-fire engine test facility. The fourth 
configuration is the flight hardware. All four configurations include the same LE-7A turbopump whose cavitation compliance and mass flow gain factor were extracted from the ground tests and were included in Figure 3. The dynamic model for these LE-7A turbopump systems incorporated the time domain equivalent of the pump transfer function including pump cavitation compliance and mass flow gain factor terms as well as the known steady pump performance characteristic. It also included lumped parameter models for the storage tank (fuel or oxidizer), the accumulator, and the valves, as well as compressible, frictional flow equations for the flows in the feedlines. The assumed boundary conditions at inlet to and discharge from these hydraulic systems were an assumed storage tank pressure and the back pressure in the combustion chamber or catchment tank. Additional, pseudopressure terms (Batchelor, 1967) were included in the flight configuration to account for the globallyimposed acceleration, a. These model equations were solved numerically in the time domain using the traditional methods of fluid transients (Wylie et al. 1993, Brennen 1994) including the method of characteristics for the feedlines. Low-level white noise pressure perturbations were injected at the pump inlet in order to provide a trigger for potential cavitation surge, should that be inclined to occur. This technique is based on the assumption that the cavitation surge (and other dynamic responses) observed in the ground-based tests are similarly triggered by random pressure noise.

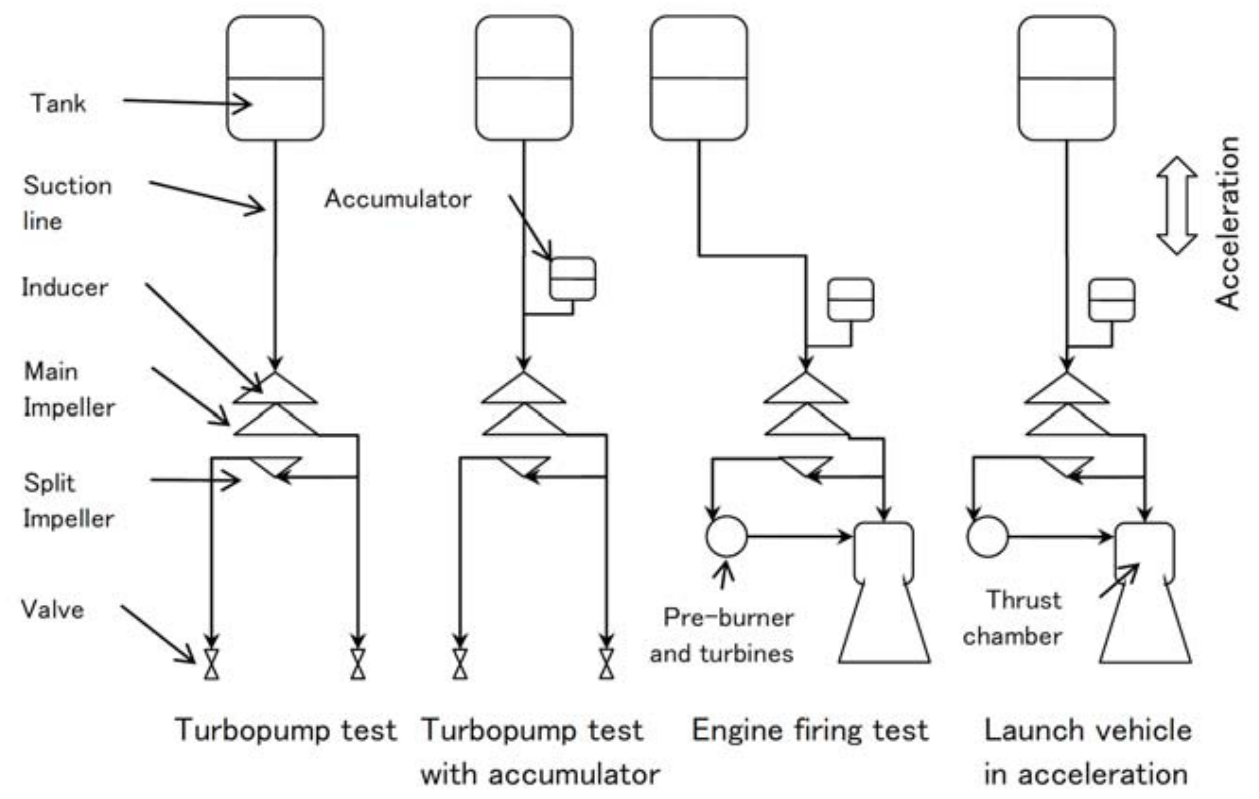

Figure 9. The four hydraulic system configurations whose dynamic responses are compared.

\section{Comparing the system response and stability}

The results that Hori and Brennen (2011) obtained for the four LE-7A test sytems are obtained are presented in Figures 10, 11, 12, 13. In the case of the first three ground-based configurations comparison is made with pressure spectra obtained during the system testing.

The calculated and measured spectra for the first configuration are shown in Figure 10. and show excellent agreement. For a cavitation number greater than 0.04, the pressure fluctuations are very small indeed. However, when the cavitation number is decreased into the range 0.033 to 0.020 , pressure fluctuations at a non-dimensional frequency of 0.22 become dominant; as described earlier this is the natural frequency of the cavitating pump and the increase occurs when there is a resonance between that natural frequency (which decreases as $\sigma$ decreases) and the third organ pipe mode of oscillation of the suction line. However, even these resonant pressure oscillations are inconsequential; for example the amplitude at the inducer discharge is less than $0.4 \%$ of inducer tip dynamic pressure. Note that the spectra also include very small pressure fluctuations at non-dimensional frequencies of 0.13 and 0.31 ; these correspond to the second and the fourth organ pipe modes of the suction line. 

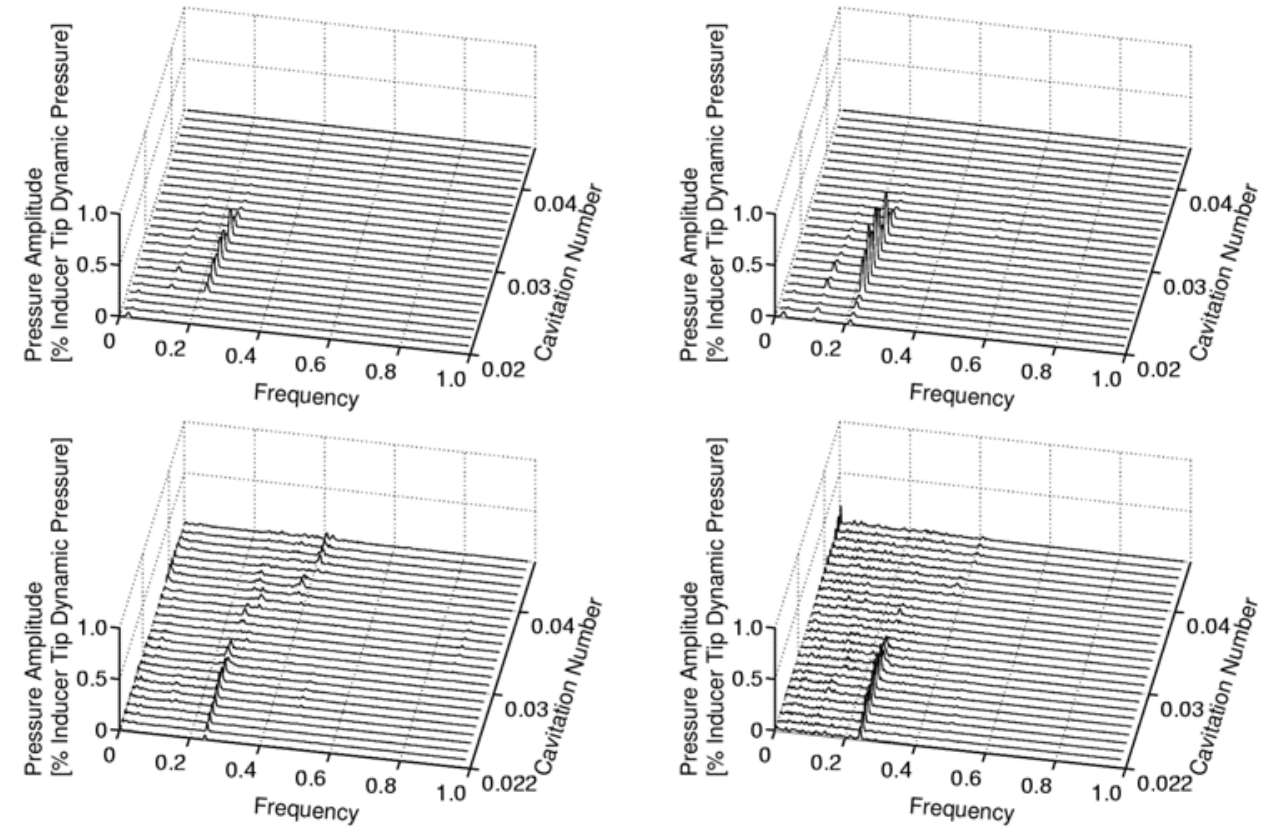

Figure 10. Model calculations (upper graphs) and test facility measurements (lower graphs) of the pump inlet pressure (left) and the inducer discharge pressure (right) from the cold test facility without an accumulator, the first configuration.

Sample results for the second configuration, a different cold-test facility with an accumulator, are presented in Figure 11. The most obvious change from the first configuration is the appearance of a natural resonant oscillation of the flow between the accumulator and the cavitation in the pump. This occurs because of the short length (and therefore small inertance) of fluid between the accumulator and the cavitation. As the cavitation number decreases and the cavitation compliance increases, the frequency of this natural cavitation surge decreases. For $\sigma>0.040$, the inducer pressure fluctuations involved are very small. But when $\sigma$ is reduced to 0.037, a double resonance occurs involving the natural frequency of the cavitating pump, the frequency of oscillating of the fluid between the accumulator and the pump and the third organ pipe mode of the feedline between the tank and the pump. This double resonance results in a sudden, substantial increase in the magnitude of the pressure oscillations. With further decrease in $\sigma$ to 0.035 , the fluctuation magnitude decreases again as the double resonance has passed. The corresponding experimental spectra exhibit good qualitative agreement with the model calculations; the higher harmonics observed in the test and which do not appear in the model calculations are probably caused by non-linear effects. However, despite this double resonance, both the tests and the calculations exhibit very small pressure oscillation amplitudes, less than $1 \%$ of inducer tip dynamic pressure and, as in the first configuration, this magnitude is inconsequential.

Spectra for the third configuration, the hot-firing engine test are shown in Figure 12. As in the second configuration, the response is dominated by a strong resonance of the fluid between the pump and the accumulator. The frequency of this resonance decreases from 0.5 to 0.2 as the cavitation number is decreased from 0.05 to 0.02 (the frequencies are higher than in the second configuration because the accumulator is much closer to the turbopump). Again, the model results appear to simulate the test data very well, matching both the frequency and the amplitude. However, the pressure amplitudes are still very small, less than $0.01 \%$ of the inducer tip dynamic pressure. Even when the peak frequency matches one of the suction line organ pipe frequencies, no large pressure oscillation magnitudes occur because the suction line in the hot-firing engine test facility is very long and the suction line resistance is large. 

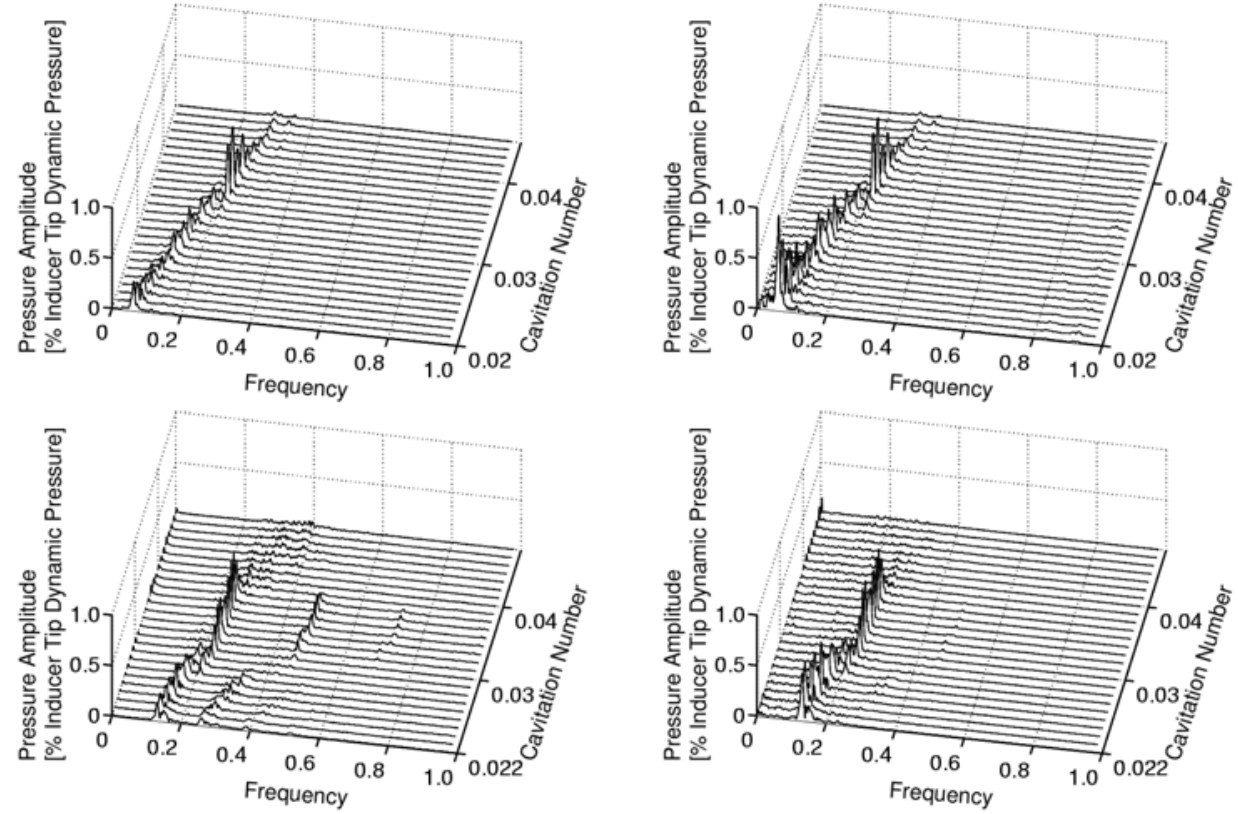

Figure 11. Model calculations (upper graphs) and test facility measurements (lower graphs) of the pump inlet pressure (left) and the inducer discharge pressure (right) from the cold test facility with an accumulator, the second configuration
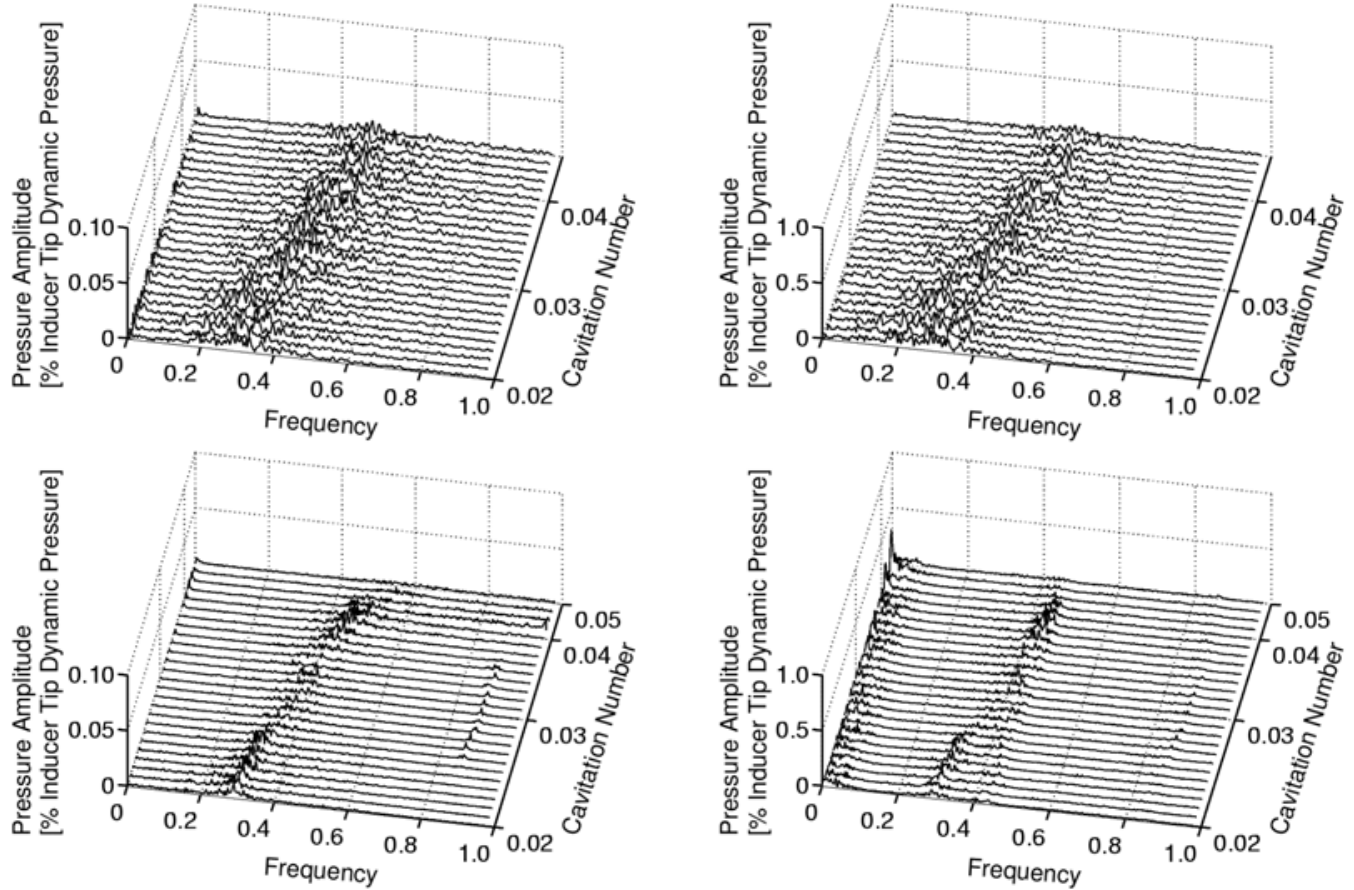

Figure 12. Model calculations (upper graphs) and test facility measurements (lower graphs) of the pump inlet pressure (left) and the inducer discharge pressure (right) from the hot-firing engine test, the third configuration. 

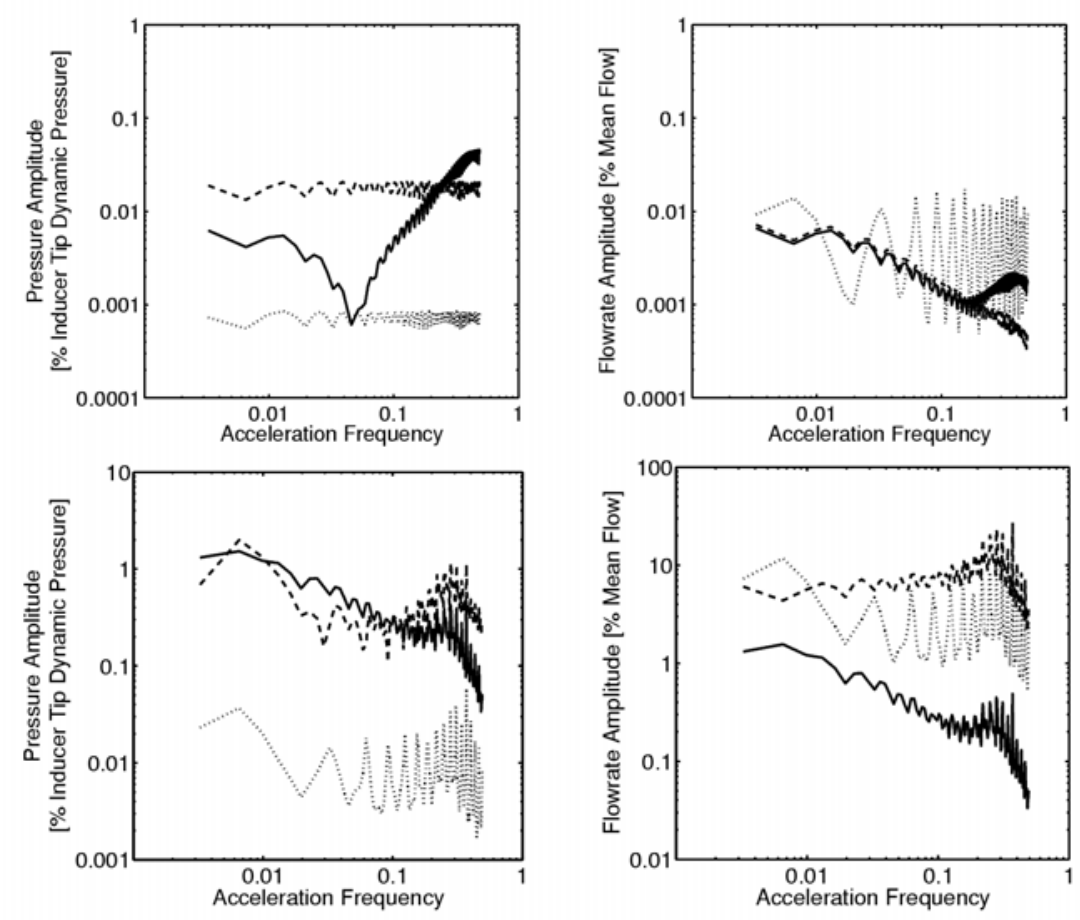

Figure 13. Model calculations for the flight configuration subject to global acceleration. Upper graphs: in the absence of pump cavitation. Lower graphs: when the pump cavitation number is $\sigma=0.02$.

Pressure amplitudes (left) and flow rate amplitudes (right) over a wide range of different oscillation frequencies and an oscillating acceleration magnitude of $0.1 \mathrm{~m} / \mathrm{s}^{2}$. Solid, dashed and dotted lines respectively present the pump discharge, inducer inlet and tank outlet quantities.

Having to some extent validated the model calculations, Hori and Brennen (2011) then turned to the flight configuration. First the response of the flight configuration without imposed acceleration was investigated and only very small pressure oscillations (less than $0.01 \%$ of inducer tip dynamic pressure) and flowrate oscillations (less than $0.01 \%$ of mean flow) were calculated. Thus, like the first three configurations, the flight configuration is very stable in a non-accelerating frame. Then the model was used to examine the response of the flight configuration in a sinusoidally accelerating frame with an acceleration amplitude of $0.1 \mathrm{~m} / \mathrm{s}^{2}$ at various non-dimensional frequencies ranging from 0 to 0.5 . The magnitude $0.1 \mathrm{~m} / \mathrm{s}^{2}$ would be characterisitic of the background excitation experienced in the rocket environment. Typical model results under non-cavitating conditions are shown in the upper graphs of Figure 13. and are similar in magnitude to the results for the ground-based calculations; the conclusion is that, in the absence of cavitation, the system response is quite muted with pressure oscillation magnitudes less than $0.05 \%$ of inducer tip dynamic pressure and flow rate oscillation magnitudes less than $0.02 \%$ of mean flow.

Finally Hori and Brennen (2011) present their key result, namely the response of the flight configuration to the same range of global oscillation (an acceleration magnitude of $0.1 \mathrm{~m} / \mathrm{s}^{2}$ for a range of oscillation frequencies), when the pump is cavitating. The lower graphs of Figure 13. present the results for the lowest cavitation number examined namely $\sigma=0.02$. It is clear that the result is a violent resonant response with amplitudes about two orders of magnitude greater than in the absence of cavitation. The pressure oscillation magnitudes are more than $2 \%$ of inducer tip dynamic pressure and the flow rate oscillation magnitudes are more than $20 \%$ of mean flow. Under these cavitating conditions, the largest flow rate magnitudes occur between the accumulator and the inducer at all frequencies and the largest pressure amplitudes occur at the inducer discharge. Thus the flow rate oscillation between the accumulator and the inducer dominates the overall response and excites the 
rest of the system like an oscillating piston. The suction line from the tank to the accumulator also plays a role, albeit a secondary role. When the frequency of the "piston" coincides with an organ pipe mode of the compressible liquid between the tank and the cavitating inducer the entire system exhibits a peak response and this happens at each of those organ pipe modes. There is also an important global response maximum near the natural frequency of the cavitating pump (0.3); at higher frequencies the response dies off rather rapidly.

Thus the model calculations demonstrate how a violent resonant response can occur in the accelerating flight environment when pump cavitation is present and that this response can occur even when all the ground tests (and the model flight calculations without cavitation) indicate a stable and well-behaved response. The difficulty of duplicating these adverse flight environments in any ground test - and therefore of examining such an adverse condition - makes accurate model calculations an almost essential design tool.

\section{Conclusion}

In concluding this review we should remark that despite significant progress in understanding the dynamics of cavitation in pumps and inducers, there is much that remains to be accomplished before an adequate pump system design procedure is completed. It is, perhaps, most useful in these concluding remarks to identify some of the most glaring gaps in our knowledge.

In terms of accomplishments we do have a reasonable data base supporting our preliminary understanding of the scaling of the dynamic transfer function with pump size, pump rotating speed (admittedly within a fairly narrow speed range), cavitation number and flow coefficient. However, most of that data is in water at roughly normal temperatures. Therefore the first deficiency is the lack of experimental data for the thermal effects on the dynamics. Thermal effects on cavitation and on the steady state performance of pumps have been extensively studied and are well known, for example, in the context of cryogenic pumps (see, for example, Brennen 1994); thermal effects in liquid oxygen are important and they are pervasive in liquid hydrogen pumps. But, apart from some preliminary tests (Brennen et al. 1982, Yoshida et al. 2009, 2011) and some very limited theoretical considerations (Brennen 1973), little is really known about the thermal effects on the dynamic characteristics of cavitating pumps. Testing in fluids other than water is very limited though the recent work of Yoshida et al. (2011) in liquid nitrogen suggests little thermal effect on cavitation surge. The lack of data is, in large measure, due to the absence of dynamic flow meters for non-aqueous environments. Electromagnetic flow meters have proved invaluable in the water tests, in part, because of their unique ability to measure the cross-sectionally integrated flow rate irrespective of axisymmetric velocity profile and, in part, because of their dynamic capability. (Electromagnetic meters for cryogenic fluids are not out of the question and should be constructively investigated). It seems likely that thermal effects could substantially dampen the dynamic characteristics and, if so, it would be valuable to confirm or refute this.

Another gap that has become evident in recent years and that has a significant impact on pump system stability is the effect of complex values for the compliance and mass flow gain factor. Though we have described above some very crude data on the phase lags for compliance and mass flow gain factor this data has very large uncertainties associated with it and we have little knowledge about how the values scale with speed or size. These effects and their uncertainty strongly suggest that a more extensive transfer function data base is needed that would not only examine the thermal effects but also extend the data to higher speeds. Such experimental investigations should also investigate the non-linear effects that obviously limit the amplitude of the cavitation instabilities and the resonant responses.

Another major gap in our current understanding has been evident for some time through the work of Rubin and others on the response of pump systems in globally oscillating environments and was particularly evident in the work of Hori and Brennen described above. There are some very real questions about the dynamic response of cavitation and of cavitating pumps subjected to translational or rotational acceleration. The only surefire way to answer these questions is to conduct experiments 
with a pump loop experiment mounted on a shaker table that can impose substantial global oscillations up to frequencies of the order of $50 \mathrm{~Hz}$ or more. Given the availability of huge shaker tables for earthquake engineering research and the known destructive consequences of instabilities such as the Pogo instability of liquid-propelled rockets, it is surprising that such experiments have not been carried out in the past.

Finally, I can anticipate that some will promote the use of computational models for cavitating flows in order to try to bridge these gaps. Though there have been some valuable efforts to develop CFD methods for cascades (see, for example, Iga et al. 2004), the problem with this suggestion is that accurate numerical treatments for cavitating pumps that will adequately represent both the nonequilibrium character of cavitation and adequately respond to flow fluctuations are still in a very early stage of development. Codes that can also handle the complex geometry and turbulence of the flow in an inducer including the tip clearance backflow are many years away. It seems clear that much progress will be needed in the development of reduced-order models for cavitation before the computational approach can produce useful, practical results.

\section{Acknowledgments}

The author wishes to acknowledge the extensive and valuable support provided by the NASA George Marshall Space Flight Center, Huntsville, AL, during much of the research discussed in this paper. I also owe a great debt to my colleague and collaborator Allan Acosta as well as to numerous students at Caltech, particularly S.L.Huang and David Braisted. The numerous constructive discussions with long time associates Loren Gross, Henry Stinson, Sheldon Rubin, Jim Fenwick, Tom Zoladz, Kenjiro Kamijo, Yoshi Tsujimoto and Shusuke Hori are gratefully acknowledged. I also appreciate the support of JAXA, the Japan Aerospace Exploration Agency, in sponsoring the visit of Shusuke Hori to Caltech.

\section{Nomenclature}

\begin{tabular}{|c|c|c|c|}
\hline & Global acceleration of the pumping system & $\mathrm{R}_{\mathrm{t}}$ & Radius of the inducer tip \\
\hline $\mathrm{C}$ & Cavitation compliance & $\mathrm{TP}_{\mathrm{ij}}$ & Pump transfer function elements \\
\hline $\mathrm{C}^{*}, \mathrm{M}^{*}$ & Quadratic compliance and mass flow gain & $\mathrm{U}_{\mathrm{s}}$ & Velocity in the cavitation zone \\
\hline & factor coefficients & $\mathrm{U}_{\mathrm{t}}$ & Velocity of the inducer tip \\
\hline $\mathrm{f}$ & Frequency [Hz] & $\phi_{1}$ & Inlet flow coefficient \\
\hline h & Inducer blade tip spacing, $2 \pi R_{t} / N$ & $\sigma$ & Cavitation number \\
\hline & $(-1)^{1 / 2}$ & & Liquid density \\
\hline$K^{\prime}, M^{\prime}$ & Bubbly flow model parameters & $\tau_{\mathrm{C}}, \tau_{\mathrm{M}}$ & Phase lags of the compliance and mass flow \\
\hline & Pump inertance & & gain factor \\
\hline $\mathrm{L}_{\mathrm{s}}$ & Length of the cavitation zone & $\omega$ & Dimensional radian frequency \\
\hline $\mathrm{m}$ & Complex fluctuating mass flow rate & $\omega^{\prime}$ & Dimensionless frequency, $\omega \mathrm{h} / \mathrm{U}_{\mathrm{t}}$ \\
\hline M & Mass flow gain factor & $\Omega$ & Frequency of rotation of the pump \\
\hline $\mathrm{N}$ & Number of main inducer blades & $\Omega_{\mathrm{p}}$ & Natural cavitation frequency of the inducer \\
\hline & Complex fluctating total pressure & $\Omega_{\mathrm{P}}^{\prime}$ & Dimensionless natural cavitation frequency of $t$ \\
\hline & Pump resistance & & he inducer, $\Omega_{\mathrm{p}} \mathrm{h} / \mathrm{U}_{\mathrm{t}}$ \\
\hline
\end{tabular}

\section{References}

[1] Ng S L and Brennen C E 1978 ASME J. Fluids Eng., 100, 166-176.

[2] Brennen C E, Meissner C, Lo E Y and Hoffman G S 1982 ASME J. Fluids Eng., 104, 428-433.

[3] Rubin S 1966 AIAA J. Spacecraft and Rockets, 3, No. 8, 1188-1195.

[4] Rubin S 1970 Prevention of coupled structure-propulsion instability (POGO) NASA SP-8055.

[5] Oppenheim B W and Rubin S 1993 AIAA J. Spacecraft and Rockets, 30, No. 3, 360-373.

[6] Tsujimoto Y, Kamijo K and Brennen C J. Propulsion and Power, 17, No.3, 636-643. 
[7] Dotson K W, Rubin S and Sako B H. 2005 AIAA J. Propulsion and Power, 21, No. 4, 619-626.

[8] Ohashi H. 1968 NASA TN D-4298.

[9] Anderson D A , Blade R J and Stevens W 1971 Response of a radial-bladed centrifugal pump to sinusoidal disturbances for non-cavitating flow NASA TN D-6556.

[10] Brennen C E. 1994 Hydrodynamics of Pumps. Oxford Univ. Press. and Concepts ETI.

[11] Brennen C E and Acosta A J 1976 ASME J. Fluids Eng., 98, 182-191.

[12] Shimura T 1995 AIAA J. Propulsion and Power, 11, No. 2, 330-336.

[13] Hori S and Brennen C E. 2011 J. Spacecraft and Rockets, 48, No.4, 599-608.

[14] Sack L E and Nottage H B 1965 ASME J. Basic Eng., 87, 917--924.

[15] Rosenmann W 1965 Experimental investigations of hydrodynamically induced shaft forces with a three bladed inducer Proc. ASME Symp. on Cavitation in Fluid Machinery, 172--195.

[16] Natanzon M S, Bl'tsev N E, Bazhanov V V and Leydervarger M R 1974 Fluid Mech., Soviet Res., 3, No.1, 38--45.

[17] Miller C D. and Gross L A 1967 A performance investigation of an eight-inch hubless pump inducer in water and liquid nitrogen NASA TND-3807.

[18] Braisted D M and Brennen C E 1980 Polyphase Flow and Transport Technology, ed. R. A. Bajura, ASME Publ., New York, 157-166.

[19] Zoladz T 2000 Observations on rotating cavitation and cavitation surge from the development of the fastrac engine turbopump 36th AIAA/ASME/SAE/ASEE Joint Propulsion Conf., AIAA-2000-3403, Huntsville, AL.

[20] Kamijo K, Shimura T and Tsujimoto Y 1994 ASME Cavitation and Gas-Liquid Flow in Fluid Machinery and Devices, 190, 33-43.

[21] Tsujimoto Y, Kamijo K and Yoshida Y 1993 ASME J. Fluids Eng., 115, No. 1, 135-141.

[22] Hashimoto T. Yoshida M, Watanabe M, Kamijo K and Tsujimoto Y 1997 AIAA J. Propulsion and Power, 13, No. 4, 488-494.

[23] Brennen C 1973 ASME J. Fluids Eng., 95, 533-541.

[24] Otsuka S, Tsujimoto Y, Kamijo K and Furuya O 1996 ASME J. Fluids Eng., 118, 400-408.

[25] Rubin S 2004 An interpretation of transfer function data for a cavitating pump 40th AIAA/ASME/SAE/ASEE Joint Propulsion Conference, AIAA-2004-4025, Florida.

[26] Brennen C E 1978 J. Fluid Mech., 89, 223-240.

[27] Cervone A, Tsujimoto Y and Kawata Y 2009 ASME J. Fluids Eng., 131, No. 4.

[28] Yonezawa K, Aono J, Kang D, Horiguchi H, Kawata Y and Tsujimoto Y 2012 International J of Fluid Machinery and Sysyem (to be piblidhed).

[29] Cervone A, Tsujimoto Y and Kawata Y 2009 ASME J. Fluids Eng., 131, No. 4.

[30] Batchelor G K. 1967 An Introduction to Fluid Dynamics Cambridge Univ. Press.

[31] Wylie E B, Streeter V L and Suo L. 1993 Fluid Transients in Systems Prentice Hall.

[32] Yoshida Y, Sasao Y and Watanabe M 2009 ASME J. Fluids Eng., 131, No. 9.

[33] Yoshida Y, Nanri H, Kikuta K, Kazami Y, Iga Y and Ikohagi T 2011 ASME J. Fluids Eng., 133, No. 6.

[34] Iga Y, Nohmi M, Goto A and Ikohagi T 2004 ASME J. Fluids Eng., 126, No. 3, 419-429. 